\title{
Benefícios do Exercício Físico sobre a Área Emocional
}

Rossane Frizzo de Godoy ${ }^{1}$

\section{Resumo}

A preocupação com o bem-estar biopsicossocial do homem tem sido tema de uma se'rie de estudos e contribuições. Esse artigo de revisão tem como objetivo básico auxiliar profissionais de diversas áreas, especialmente as ligadas as $\mathbf{C i}$ ências do Movimento Humano, a compreenderem, de maneira ampla, o impacto dos problemas relacionados à saúde mental e suas manifestações mais comuns na sociedade atual: a ansiedade, a depressão, alterações de auto-imagem e auto-estima. Procurando oferecer maneiras alternativas para se prevenir e auxiliar no tratamento desses problemas. $O$ exercício físico, nesse contexto, tem se mostrado eficaz para modificá-las.

DESCRITORES: Ansiedade, depressão, auto-estima, auto-imagem, exercício.

\section{Abstract}

Searchingfor human biopsychosocial well being has been the major objective of an impressive number of studies. This article reviews the current knowledge aboutthe impact of anxiety, depression and selfesteem on the individual. Besides, it describes some insights to preveni and to treat these disturbs. In this context, the practice of a physical exercise may be efficient to attain both objectives.

Key words: anxiety, exercise.

\section{Introdução}

0 National Institute of Mental Health (Regier et al., 1984) através de uma investigação, demonstrou, já em meados da década de 80 , que $20 \%$ da população adulta americana sofre de alguma forma de transtorno mental.

No Brasil, os transtornos de ansiedade e depressão são responsáveis por uma enorme sobrecarga para os serviços de saúde, acometendo ao longo da vida, respectivamente, $20 \%$ e $30 \%$ da população, segundo Ito (1998).

Lawlor e Lazare (1989) referem que esses números são significativamente aumentados se forem observadas populações que também apresentam transtornos clínicos, dentre as quais destacam-se as doenças cardiovasculares, endócrinas e metabólicas, doenças inflamatórias, hematológicas, gastrintestinais, infecciosas e pulmonares.

A ansiedade pode ser considerada como universal, trata-se de um fenômeno eminentemente humano, caracterizado por uma antecipação temerosa de um acontecimento futuro desagradável. E uma emoção experimentada pela pessoa como uma ameaça tanto física quanto psíquica que provoca uma reação de alerta, conforme o DSM-IV (1995) e Kaplan e Sadock (1984).

A ansiedade pode manifestar-se em vários momentos da vida cotidiana, sofrendo variações de intensidade e duração, dependendo das circunstâncias.

Rojas (1997) utiliza diferentes denominações para a ansiedade. A ansiedade positiva refere-se a um estado de ânimo presidido pelo interesse, a curiosidade, o afã de conhecer.

Na filosofia existencialista, o homem é compreendido como um constante vir-a-ser, isso por si só abarcaria um certo nível de tensão, uma vez que, uma característica da existência humana é estar sempre a caminho, situada frente a novas possibilidades de decisão desta- 
ca Zilles (1988). De acordo com Heidegger (1991), esse tipo de tensão também denominada angústia existencial, ocorre no homem pelo simples fato deste existir, pois provém da inquietude frente à vida, colocando o homem frente a seu destino, frente à morte, frente as suas escolhas.

Por outro lado, segundo Hetem (1997), a ansiedade pode ser negativa, patológica quando a freqüência e a intensidade das manifestações ansiosas vierem, por sua amplitude, perturbar de modo mais ou menos notável a vida do indivíduo, sua adaptação ao mundo, seu equilíbrio afetivo e intelectual, havendo, portanto, um comprometimento no funcionamento psicossocial da pessoa.

Spielberger, Gorsuch e Lushene (1979), falam na ansiedade-estado e na ansiedade-traço. A ansiedadeestado refere-se a uma reação ou resposta emocional, que é evocada por um indivíduo que percebe uma situação particular como sendo potencialmente perigosa ou ameaçadora para ele, a despeito da presença ou ausência de um perigo real. Portanto, a ansiedadeestado varia de acordo com a situação vivida pela pessoa. Já a ansiedade-traço, por outro lado, é uma característica relativamente estável da personalidade de cada indivíduo.

Ito (1998) comenta que a tristeza é uma reação normal que pode ocorrer em muitos momentos da vida, principalmente frente a situações que envolvam perda ou decepção. Esse estado de humor não deve ser confundido com a depressão clínica, que traz conseqüências importantes para a vida da pessoa, interferindo em sua capacidade de trabalhar, divertirse, relacionar-se e dormir.

Um aspecto básico da depressão, para EY, Bernard e Brisset (1981), é a observação do abatimento do humor, que se torna triste. Aliando-se à presença da inibição e do sofrimento moral.

A inibição é considerada um tipo de freio utilizado pelo paciente, que o leva a fechar-se em si mesmo e a fugir dos outros. Esse aspecto é, também, observado na área do pensamento. O paciente sente dificuldade para pensar, apresenta distúrbios de memória, fadiga psíqui- ca e também lentidão na atividade motora.

O sofrimento moral exprime-se em sua forma mais elementar de autodepreciação, auto-acusação e autopunição.

Autores como Beck, Rush, Shaw e Emery (1997) estimaram que pelo menos $12 \%$ da população adulta teve ou terá um episódio de depressão de severidade, que justifique tratamento.

Dentro dessa perspectiva preocupante, poder-se-ia pensar de que maneira esses episódios de ansiedade e depressão seriam prevenidos ou minorados. $\mathrm{Na}$ área da Psicologia Esportiva, vários estudos têm sido realizados com o objetivo de compreender os efeitos e os benefícios do exercício e do esporte sobre a área emocional, procurando observar as inter-relações existentes entre a prática de uma atividade física e o bem-estar emocional do ser humano.

\section{Exercício Físico e Saúde.}

Elbas e Simão (1997) salientam que há menos de um século, o exercício físico não era algo que as pessoas fizessem para ficar em forma. O exercício fazia parte da rotina das pessoas: cortar lenha, fazer longas caminhadas para visitar amigos, etc.

OISSP (1992) destaca que, durante o século XX ocorreu uma redução substancial na frequiência e na intensidade com que as pessoas se engajavam em atividades físicas constantes e equilibradas.

Com as mudanças e facilidades decorrentes do progresso, observou-se uma significativa modificação no comportamento do ser humano. $\mathrm{O}$ aumento da carga horária de trabalho, a mudança de atividades profissionais, exigindo que se fique sentado praticamente todo o dia, a obesidade, o estresse e o sedentarismo foram levando a uma mudança de hábitos que, conseqüentemente, se refletiram sobre a saúde do Homem.

Cooper (1979) que em 1968 lançou o livro "Aptidão Física em Qualquer Idade", denotou uma preocupação evidente de se buscar uma vida mais saudável, popula- 
rizando os conhecimentos e práticas do exercício, aplicado à Medicina Preventiva e à Reabilitação em geral.

Com o aumento de informações na área, cada vez mais pessoas procuram estudar os beneficios que a atividade física pode ocasionar na saúde, tanto física quanto mental, destacam Araújo Filho e Gomes (1995).

O American College of Sport Medicine (1986), divide os exercícios em três categorias:

a) Cardiorrespiratórios ou resistência aeróbia

b) Força e resistência muscular

c) Flexibilidade

Os programas de exercícios físicos direcionados a promoção da saúde devem utilizar, segundo Cossenza (1996), exercícios que estimulem, predominantemente, o sistema cardiorrespiratório. O sistema respiratório apresentará maior facilidade para transportar e aproveitar o oxigênio, com um custo energético menor, aumentando o débito cardíaco, fortalecendo o miocárdio e regulando a pressão arterial (Lima, 1998).

Segundo a ACMS (1986) para se conseguir os beneficios de saúde propiciados pelo exercício, a pessoa precisa somente participar de uma atividade física leve a moderada. A caminhada tem demonstrado imensos benefícios para a saúde, podendo ser praticada em qualquer idade, sendo ideal para pessoas idosas.

A força muscular e a flexibilidade, nas opiniões de Phillips, Pruitt e King (1997), contribuem muito para se ter uma vida mais saudável e independente, principalmente nas idades mais avançadas.

Cossenza e Carvalho (1997) ressaltam que a finalidade dos exercícios com força ou resistência muscular, em pessoas idosas, objetiva evitar quedas, evitar fraturas, contribuindo para uma melhora postural e aumento da força para a realização das atividades de vida diária.

Achour Jr. (1998) reforça que a estrutura de programas de flexibilidade, para a promoção da saúde e bem-estar, deve implementar exercícios de alongamento, a fim de evitar e/ou eliminar encurtamentos musculares e lesões ocasionadas por esforços exigidos no trabalho ou para aliviar tensões musculares.

Outras vantagens propiciadas pelo exercício fisico regular, citadas por Ramos (1997) são:

- Melhora na estabilidade articular;

- Aumento da massa óssea;

- Aumento do colesterol HDL;

- Diminuição da freqüência cardíaca de repouso;

- Diminuição do risco de arteriosclerose, varizes, acidente vascular cerebral, lombalgia;

- Aumento da flexibilidade, força, resistência aeróbia e anaeróbia;

- Facilitação da correção de vícios posturais;

- Facilitação da mecânica respiratória;

- Diminuição do estresse psicológico.

Elbas e Simão (1997) referem ainda:

- Aumento da habilidade do sistema nervoso mandar e receber mensagens;

- Aumento da resistência psicofísica, aumentando a performance em atividades físicas e trabalhos mentais;

- Melhoria da sensação de bem-estar;

- Redução da fadiga e ansiedade;

- Aumento da auto-estima.

\section{Benefícios do Exercício Físico sobre a Área Emocional}

Muitos estudos vêm sendo realizados para procurar compreender quais são os efeitos do exercício físico sobre a área emocional.

Cossenza e Carvalho (1997) sugerem que a atividade fisica pode ser um meio alternativo excelente para descarregar ou liberar tensões, emoções e frustrações, acumuladas pelas pressões e exigências da vida moder- 
na.

Uma pesquisa realizada por Lobstein, Mosbacher e Ismail (1983) demonstra que a depressão foi a variável psicológica mais importante para distinguir grupos de pessoas de meia idade sedentárias de grupos de pessoas que praticavam atividade física.

Francis e Carter (1982), também comparando grupos de homens sedentários com grupos de homens que se exercitavam com regularidade, referem que os níveis de ansiedade, hostilidade e depressão do grupo que se exercitava eram muito mais baixos do que os níveis nos sedentários.

Doyne (1987) mostra que a falta de exercício físico e um fator importante para o aparecimento de sintomas de ansiedade e depressão, o que vem a corroborar os resultados das pesquisas acima mencionadas.

Cooper (1979), com o intuito de buscar uma medicina mais preventiva, fez, já na década de 70, um programa de exercícios aeróbios com 60 homens de meia idade, atribuindo a prática do exercício físico a melhoria na auto-imagem dessas pessoas.

Scully, Kremer, Meade et al. (1998) demonstraram os efeitos positivos do exercício sobre os níveis de ansiedade e depressão, diminuindo-as e, melhorando a auto-estima, o autoconceito e a imagem corporal.

Lane e Lovejoy (2001) estudaram os efeitos do exercício sobre as mudanças de humor e concluíram que o exercício está associado com as melhoras de humor das pessoas que tinham sintomas depressivos antes de iniciarem os exercícios. Resultados semelhantes também foram encontrados por Berger, Owen, Motl et al. (1998).

Num estudo com populações não clínicas, realizado por Ross e Hayes (1988), foi encontrada uma correlação j positiva entre o exercício físico e o bem-estar, evidenciando que o exercício diminui os sintomas de depressão e ansiedade.

Penninx, Rejeski, Pandya et al. (2002) realizaram uma comparação dos efeitos de exercícios aeróbicos e exercícios de resistência com grupos de idosos com altos e baixos níveis de depressão. A redução nos níveis de depressão ocorreu tanto nos idosos com níveis elevados, quanto nos idosos com níveis baixos de depressão.

Estudos de Long e Haney (1988), McÂuley (1996) e Long e Van-Stavel (1995) têm demonstrado uma redução significativa nos níveis de ansiedade através da prática do exercício físico.

Barke e Smith (1985), numa investigação com crianças, também provaram que houve uma redução importante da ansiedade nas crianças que estavam inicialmente muito ansiosas.

Pesquisando estudantes universitárias, Hayden e Allen (1984) perceberam uma associação significativa entre a atividade aeróbia e a diminuição dos níveis de ansiedade e depressão.

Outro estudo, de Bartlewski (1996) com populações também universitárias, além de encontrar redução da ansiedade, concluiu que o exercício aeróbio produz um aumento nos mVeis de auto-imagem.

McCann e Holmes (1984), compararam 43 mulheres depressivas subdivididas em três grupos: $\mathrm{I}^{\mathrm{O}}$ - com programa de exercícios aeróbios; $2^{\circ}$ - com sessões de relaxamento e $3^{\circ}$ - um grupo sem tratamento. Encontrou um decréscimo nos níveis de depressão só no grupo que praticava exercícios aeróbios.

Wykoff (1993), além da redução da depressão, confirmou que o exercício físico melhora o humor e a condição da pessoa para reagir frente a estressores psicossociais.

Numa investigação de Morgan, Roberts, Brand et al. (1970) com 101 homens em programas de exercícios diferenciados, englobando natação, caminhada e corrida, com duração de seis semanas de exercício, constatou-se que estes não produziram uma redução significativa nos índices de depressão.

No entanto, outra pesquisa realizada com quatro grupos de homens de 50 a 80 anos, com duração de 12 semanas, provou uma redução nos níveis de depressão, (Vallianti e Asu, 1985). 
Godoy, Becker Jr., Godoy et al. (2001), estudaram o efeito do exercício físico sobre os níveis de ansiedade, depressão e autoconceito de 30 pacientes com Doença Pulmonar Obstrutiva Crônica (DPOC), avaliados nas formas de pré e pós teste. Os pacientes foram randomizados em dois grupos, o grupo experimental e o grupo controle. A duração do programa de atividades com ambos os grupos foi de 12 semanas.

Os pacientes do grupo experimental tiveram as seguintes atividades: duas sessões semanais de exercícios físicos, incluindo trabalho com membros superiores, inferiores e exercício aeróbico, através do uso de esteira ergométrica; uma sessão de psicoterapia individual; duas sessões de fisioterapia respiratória. O grupo controle cumpriu as mesmas etapas do grupo experimental, no entanto não realizou as sessões de exercício.

Ambos os grupos apresentaram importante e significativa diminuição da ansiedade e depressão, aumento nos níveis de autoconceito e melhora no desempenho no teste de 6 minutos de caminhada. Não foram observadas diferenças estatisticamente significantes na comparação entre os grupos. A melhora nos índices não pode ser atribuída ao fator exercício.

Gayle, Spliter, Karper et al. (1988) num estudo com pacientes com DPOC, concluem que o exercício teve um impacto muito pequeno sobre os níveis de ansiedade e depressão, embora os pacientes referissem melhora. Resultados semelhantes também foram encontrados por White, Rudkin, Ashley et al. (1997).

Emery, Hauck, Schein et al. (1998) comprovou a diminuição da ansiedade dos pacientes com DPOC. Esta diferença é explicada pelo autor como sendo decorrência da carga relativamente intensa de exercícios praticados pelos pacientes desse grupo. No entanto, não houve uma diminuição maior da depressão nos pacientes praticantes de exercício.

Um aspecto importante ressaltado por Becker Jr (2000), é que, para se observar os efeitos do exercício e do esporte sobre a área emocional, é necessário que se respeite um tempo de prática, variando entre 4 e 20 semanas. A intensidade com que o exercício físico é realizado também é um fator que interfere no tipo de respos- ta emocional observada. Krause, Goldenhar, Liang et al. (1993), ressaltam que, quanto mais freqüente é a prática de exercício, menor é a presença de transtornos psicológicos e muitos agentes estressores tendem a reduzir sua força na medida em que o hábito do exercício físico aumenta.

Pesquisas de Morgan (1984 e 1985) demonstraram que a atividade fisica vigorosa diminui efetivamente os níveis de ansiedade e depressão.

Steptoe e Cox (1988) encontraram resultados diferentes, evidenciando que exercícios de alta intensidade aumentam os níveis de ansiedade, tensão e fadiga. As mudanças positivas de humor foram observadas somente nos grupos de pessoas que realizavam exercícios físicos de baixa intensidade.

Um estudo posterior de Steptoe et al. (1989) concluiu que o exercício moderado também leva a um aumento da capacidade de lidar com o estresse, diminuindo a ansiedade, a depressão e a tensão.

\section{Teorias que Explicam os Benefícios da Atividade Física sobre a Área Emocional}

Não se pode afirmar exatamente qual é o mecanismo de ação do exercício físico sobre a área emocional. Algumas teorias vêm sendo propostas para procurar explicitar esses fatores.

As hipóteses explicativas podem ser divididas em dois grandes mecanismos, conforme North, McCullager e Tran (1990):

\section{Mecanismos Psicológicos:}

a) Hipótese Cognitivo-Comportamental postulada a partir da concepção do modelo de depressão de Beck que propõe:

- a presença automática de pensamentos negativos;

- a presença sistemática de erros lógicos,

- a presença do esquema depressogênico 
O exercício teria o poder de quebrar essa espiral depressiva, através da substituição dos sentimentos e pensamentos negativos por sentimentos e pensamentos positivos.

Sime (1984) refere-se a essa hipótese dizendo haver uma melhoraria na auto-eficácia da pessoa.

b)Hipótese da Interação Social. Hipótese que seria explicada a partir do sentimento de prazer da interação grupai e do reforço social recebido pelas pessoas que praticam o exercício físico.

c) Hipótese da Distração de Sachs. A prática da atividade fisica proporcionaria uma distração das preocupações do dia -a-dia.

\section{Mecanismos Fisiológicos e Bioquímicos:}

a) Hipótese do aumento da aptidão cardiovascular. O efeito antidepressivo do exercício poderia ser mediado pelo nível de preparo cardiovascular (aeróbio). Essa hipótese tem sido proposta desde que uma relação correlacionai negativa foi achada entre o nível de capacidade aeróbia e a depressão. No entanto, os efeitos antidepressivos do exercício iniciam-se nas primeiras semanas de tratamento, antes que os indivíduos apresentem uma mudança na capacidade cardiovascular.

b) Hipótese das Aminas. Três neurotransmissores explicariam o efeito antidepressivo do exercício, são eles: serotonina, dopamina e norepinefrina. Algumas evidências indicam que indivíduos deprimidos apresentam um decréscimo na produção dessas aminas metabólicas. O exercício teria o poder de estimular o aumento da produção desses neurotransmissores.

c) Hipótese das Endorfinas. As endorfinas são substâncias produzidas no organismo e possuem qualidades capazes de reduzir a dor e produzir um estado de euforia.

North, McCullager e Tran (1990) referem que o exercício físico prolongado pode ativar o sistema de opióídes e aumentar a produção dessas substâncias.
Becker Jr. (2000) acrescenta, ainda outras três hipóteses:

d) Hipótese do Efeito tranqüilizador, obtido através do aumento da temperatura corporal,

e) Hipótese do aumento da atividade adrenal, através do exercício aumentam as reservas de esteróides que combatem o estresse.

f) Hipótese da descarga da tensão muscular, através do relaxamento promovido pelas contrações e descontrações do aparelho muscular.

\section{Conclusão}

Dentro de todas essas possibilidades abordadas que procuram explicar os efeitos antidepressivos do exercício, percebe-se que não existe um consenso entre os autores para sustentar uma única hipótese.

As diferenças de resultados foram observadas principalmente quando os grupos de pesquisas envolveram pacientes clínicos.

Apesar das discordâncias observadas, acreditamos que a prática de uma atividade física regular seja indicada para pessoas de todas as idades. Seja através de uma ação preventiva, em pessoas saudáveis ou terapêutica, em pessoas doentes.

Apesar das perdas funcionais, orgânicas e mentais, que fazem parte de um envelhecimento sadio, o exercício propicia ao idoso, auto-suficiência para realizar as atividades de vida diária e condições de manter uma relação social com o meio que o rodeia.

E importante salientar que, embora alguns casos de pessoas com depressão grave tenham reduzido seus níveis de depressão com a prática do exercício, o tratamento psicoterápico e/ou a farmacológico podem ser associados.

Novas pesquisas e contribuições continuam sendo indicadas para explicitar melhor as inter-relações entre a prática de uma atividade física e a melhora emocional que ocasiona. Apesar disso, acreditamos que os efeitos 
do exercício e do esporte sobre a área emocional são encarados de maneira bastante positiva, pois apresentam como resultados a possibilidade de:

- Reduzir a ansiedade e a depressão;

- Melhorar o autoconceito, a auto-imagem e a autoestima;

- Aumentar o vigor;

- Melhorar a sensação de bem-estar;

- Melhorar o humor;

- Aumentar a capacidade de lidar com estressores psicossociais,

- Diminuir os estados de tensão;

\section{Referências}

ACHOUR JR, A. Flexibilidade: teoria e prática. Londrina: Atividade Física e Saúde, 1998.

AMERICANCOLLEGE OF SPORT MEDICINE. Guidelinesfor exercise test and prescription. Philadelphia: Lea Febiger, 1986.

ARAÚJOFILHO, N. P.; GOMES, A. C. Cross training. uma abordagem metodológica. 2. ed., Londrina: Centro de Informações Desportivas, 1995.

BARKE, M, SMITH, R. G. Alteration in anxiety of children after exercise and resl American Corrective Therapy Journal, San Diego, v. 39, n.4, p. 90-94, 1985.

BARTLEWSKI, P. Effects of aerobic exercise on the social physique anxiety and body esteem of female college students. Woman in Sport and Physical ActivityJournal. Texas, v.5,n.2,p. 49-62,1996.

BECK, A. T., RUSH, A. J., SHAW, B. F., EMERY, C. Terapia cognitiva da depressão. Porto Alegre: Artes Médicas, 1997.

BECKER JR., B. Psicologia do exercício \& esporte. Porto Alegre: Nova Prova, 2000.

BERGER, B. G, OWEN, D. R, MOLT, R. W. e PARKS, L Relationship between expectancy of psychological benefits and mood alterations injoggers. International Journal of Sport Psychology. v.29, p.1-16,1998.

COOPER, K. Saúde total. 2. ed. Rio de Janeiro: Entrelivros Cultural, 1979.

COSSENZA,C. E; CARVALHO, N. Personal training para grupos especiais. Rio de Janeiro: Sprint, 1997.

DOYNE, EJ.; OSSIP-KLEIN, D.J.; BROWMAN, E. D.; McDOUGALL-WLSOM, L. B.; NEIMAYER, R. A. Running versus weight lifting in the treatment of depression. Journal of Consulting and Criticai Psychology, v.55,748-754,1987.

DSMHV -MANUALDIAGNÓSTICOEESTATÍSTICODOS

TRANSTORNOS MENTAIS 4 ed. Porto Alegre: Artes Médicas, 1995.

ELBAS, M; SIMÃO; R. Em busca do corpo: exercícios, alimentação e lesões. Rio de Janeiro: Shape, 1997.

EMERY, C; HAUCK, E R; SCHEIN, R. L; MACINTYRE, N. R. Psychological and cognitive outcomes of a randomized trial of exercise among patients with chronic obstructive pulmonary disease. Health Psychology, Washington, v.17, n.3,23240,1998.

EY, E; BERNARD, P.; BRISSET, C. Manual de psiquiatria. Rio de Janeiro: Masson, 1981.

FRANCIS, K. T.; CARTER, R. Psychological characteristics of joggers. Journal ofSports Medicine and Physical Fitness., v.22, n.3, p.386-91,1982.

GAYLE, R; SPITLER, D. L; KARPER, W. B.; IAGER; R M; RICE, S. N. Psychological changes in exercising COPD patients. International Journal ofRehabilitation Research. Heidelberg, v.1l, n.4, p.335-42, 1988.

GODOY, R. F.; BECKERJR. B.; GODOY, D. V.; VACCARI, P. E MICHELLI, M. El efecto dei ejercicio sobre los niveles de ansiedad, depresión y autoconcepto de pacientes con dolenciapulmonar obstructiva crônica. Revista de Psicologia dei Deporte, Universitat de les Illes Balears. v.10, n.2, p.267278,2001 .

HAYDEN, R. M; ALLEN, G. J. Relationship between aerobic exercise, anxiety, and depression: convergem validation by knowledgeable informants. Journal of Sports Medicine and Physical Fitness. Torino, v.24, n.1,p.69-74,1984.

HEIDEGGER, M. Conferências e escritos filosóficos. 4. ed. 
São Paulo: Nova Cultural, 1991.

HETEM, L. A. B. Diagnóstico diferencial dos transtornos de ansiedade. In: HETEM, L. A., GRAEFF, F. G. Ansiedade e transtornos de ansiedade. Rio de Janeiro: ECN, p. 228-247, 1997.

\section{ISSP-INIERNATIONALSOAETYOF SPORTPSYCHOLOGY.}

Physical activity and psychological benefits: aposition statement. International Journal of Sport Psychology, Roma, v.23, p.86-91,1992.

ITO, L Terapia cognitivo comportamental para transtornos psiquiátricos. Porto Alegre: Artes Médicas, 1998.

KAPLAN, H.; SADOCK, B. Compêndio de psiquiatria dinâmica. Porto Alegre: Artes Médicas, 1984.

KRAUSE, N.; GOLDENHAR, L; LIANG, J.; JAY, G. Stressand exercise amongjapanese elderly. Social Science and Medicine, v.36, n.11, p.1429-1441,1993.

LANE, A. M; LOVEJOY, D. J. The effects of exercise on mood changes: the moderating effect of depressed mood. Journal o/Sports Medicine and Physical Fitness. v.41,n.4, p.539545,2001 .

LAWLOR, T. e LAZARE, A Anxiety. In: LAZARE, A. Outpatient psychiatry. Diagnosis and treatment, 2. ed. Baltimore, Williams \& Wilkins, 1989, p. 246-251.

LOBSTEIN, D. D.; MOSBACHER, B. J.; ISMAIL, A. H.

Depression as a powerful discriminator between physically active and sedentary middle-aged men. Journal of Psychosomatic Research, v.27, n.1, p.69-76,1983.

LONG, B. C, HANEY, C. J. Long-termfollow-up ofstressed workingwoman-. a comparison ofaerobic exercise and progressive relaxation. Journal of Sport and Exercise Psychology. Champaign, v.10, n.4, p.461-470,1988.

LONG, B. C; VAN-STAVEL, R. Effects of exercise training on anxiety: a meta analysis. Journal Of Applied Sport Psychology. Lafayete, v.7, n.2, p.167-189,1995.

McAULEY, E. Acute exercise and anxiety reductiom does the environment matter? Journal of Sport and Exercise Psychology, Champaign, v.18, n.4 p.408-419,1996.

McCANN, L, HOLMES, D. S. Influence ofaerobic exercise on depression. Journal of Personality and Social Psychology.
Washington, v.46, n.5, p.1142-1147,1984.

MORGAN, W. P. Physical activity and mental health. American Academy of Physical Education Papers, Champaign, v.17, p. 132-45,1984.

Affective beneficence of vigorous physical activity. Medicine and Science in Sports and Exercise. v.17, p.94-100, 1985.

MORGAN, W. P., ROBERTS, V. A., BRAND, F.R. et al. Psychological effects of chronic physical activity. Medicine and Science in Sports. v.2, n.4, p.213-217,1970.

NORTH, T. C, McCULLAGER, .P., TRAN, Z V. Effects of exercise on depression. Exercise and Sport Science Reviews, v.18, p.379-415, 1990.

PENNINX, B. W., REJESKI, W. J. PANDYA, J., MLLER, M E., DI BARI, M. APPLEGATE, W. B. PAHOR, M. Exercise and depressive symptoms: a comparison ofaerobic and resistance exercise effects on emotional and physical function in olderpersons with high and low depressive symptomatology. Journal of Gerontology Psychology Science, v.57, n. 2, p.124 132,2002

PHILLIPS, W. T. PRUITT, L, KING, A. C. Estilo de vida ativo. Recomendações atuais. Rio de Janeiro: Sprint Magazine, v.90, p.26-33,1997.

RAMOS, A. T. Atividade fisica- diabéticos, gestantes, terceira idade, crianças, obesos. Rio de Janeiro: Sprint, 1997.

REGIER, D. A, MYERS, J. K., KRAMER, M ROBINS, L. N., BLAZER, D. G., HOUGH, R. L, EATON, W. W. e LOCKE, B. Z. The epidemiologic catchment area program. Archives of General Psychiatry, 41, p.934-41, 1984.

ROJAS, E. A ansiedade. Como superar o estresse, as fobias e as obsessões. São Paulo: Ed Mandarim, 1997.

ROSS, C, HAYES, D. Exercise and psychological well being in the community. Baltimore: American Journal of Epidemiology, v.127, n.4, p.762-771,1988.

SCULLY,D., KREMERJ., MEADE, M M, GRAHAM, R, DUDGEON, K. Physical exercise and psychological wellbeing. A Criticai Review. British Journal of Sport Medicine. v.32, n.2, p.111-120,1998.

SIME, W. E. Psychological benefits of exercise training in 
the health individual. In: Matarazzo et al. Behavioral health. A handbook of health enhancement at disease prevention. Wiley: New York, p. 488-508,1984.

\section{SPIELBERGER, C. D, GORSUCH, R. L, LESHENE, R. E.}

Inventário de ansiedade traço-estado. IDATE. Rio de Janeiro: CEPA, 1979.

STEPTOE, A., COX, S. Acute effects ofaerobic exercise on mood. Health Psychology. New York, v.7, n.4, p.329-40, 1988.

STEPTOE, A et al. The effects of exercise training on mood and perceived coping ability in anxious adultsfrom the general population. Journal of Psychosomatic Research. New York, v.33, n.5, p.537-547,1989.

VAILIANT, P.M., ASU, M. E. Exercise and its effects on cognition and physiology in older adults. Journal of Perceptual and Motor Skills. Missoula, v.61, n.3, p.10311038,1985 .

VAN-ANDEL, G. E., AUSTIN, D. R. Physical fitness and mental health: a review ofliterature. Adapted Physical Activity Quarterly. Champaign, v.1,n.3, p.207-220,1984.

WHITE, R. J, RUDKIN, S. T, ASHLEY, I., STEVENS, V. A. e BURROWS, S. Outpatient pulmonary rehabilitation in severe chronic obstructive pulmonary disease. Journal of the Royal College of Physicians of London. v.31, n.5, p.541-545,1997.

WYKOFF, W. The psychological effects of exercise on nom clinical population of adult women. Occupational Therapy in Mental Health. New York, v.12, n.3, p.69-106,1993.

ZILLES, U. Gabriel Mareei e o existencialismo. Porto Alegre: Co-edição PUCRS, Livraria Editora Acadêmica, 1988.

\section{Notas}

'Especialista em Psicoterapia-PUC

Mestre em Ciências do Movimento Humano-UFRGS

Psicóloga do Programa de Reabilitação Pulmonar do Ambulatório Central-Centro de Ciências Biológicas e da Saúde da Universidade de Caxias do Sul.

ENDEREÇOCOMERCIAL:

Rua Gen. Arcy da Rocha Nóbrega, 401/204. Caxias do Sul. CEP-95040-000.

ENDEREÇOPARACORRESPONDÊNCA:

Av. Rio Branco, 209/103. Caxias do Sul.

CEP-95010-060.

Recebido: 17/04/02

Revisado: 10/06/02

Aceito: 01/07/02 
